\title{
GROUND SHALLOW DONOR STATE OF III-V COMPOUNDS FOR ARBITRARY MAGNETIC FIELD
}

\author{
A. Obuchowicz and J. Wlasak \\ Institute of Physics, Technical University of Wroclaw \\ Wybrzeże Wyspiańskiego 27, 50-370 Wrocław, Poland
}

\begin{abstract}
Theory of shallow donor in III-V semiconductors in the presence of magnetic field is reinvestigated. Considerations are performed within the 3-level Kane model. In order to avoid singularities caused by the Coulomb potential we divide it into short- and long-range components. The latter one is the slowly varying potential and contrary to the Coulomb potential it satisfies all demands for envelope function equation. The short-range part is contributing to a chemical shift. Calculation of energies of two possible spin states of ground donor level are then performed using variational method.
\end{abstract}

PACS numbers: 78.20.Ls, 61.50.Em, 71.55.-i

The use of three-level model (TLM) [1-3] to solve the problem of donor states in III-V semiconducting compounds in the presence of magnetic field began from the work by Larsen [4]. He obtained energies of ground and one excited states using envelope trial functions (ETF). His theory was simplified by Zawadzki and Własak [5] in order to obtain higher excited states and developed by Trzeciakowski et al. [6]. All above theories are suitable for restricted range of magnetic field, Larsen's for low-field limit, next two for high-field limit. Obuchowicz and Własak [7] have presented calculations for shallow donor in zinc-blende semiconductors suitable for whole range of magnetic field.

Previous theoretical works [4-7] have treated Coulomb potential of impurity as slowly varying in coordinate space, extending over many unit cells. Unfortunately, this assumption leads to infinite terms in equation for expectation value of energy within variational procedure (see Eq. (6)). Therefore, in order to obtain $s$ symmetry ground impurity state, all previous papers had to neglect these terms. The purpose of this paper is to give a theoretical description of magnetodonors which remains $s$ symmetry function for ground state without neglecting any singular terms.

In order to achieve this the Coulomb potential of donor is divided into two parts

$$
V(r)=V_{r}(r)+V_{s}(r),
$$


using the identity equation

$$
\frac{1}{r}=\frac{a}{r(r+a)}+\frac{1}{r+a} \text {. }
$$

The strongly varying part $V_{r}$ is related to chemical shift. $V_{s}$ is treated as slowly varying in the unit cell. The constant $a$ is the fitting parameter.

The problem of donor in III-V compounds under a magnetic field is described now by the set of eight equations [5]:

$$
\sum_{i=1}^{8}\left[D_{i j}+\delta_{i j}\left(V_{s}-E\right)\right] f_{j}=0,
$$

where $D_{i j}$ is the matrix of TLM effective Hamiltonian calculated with LuttingerKohn amplitudes taken in the form of [5]. $D_{i j}$ include matrix elements of strongly varying part of Coulomb potential

$$
\begin{aligned}
& A=\left\langle S\left|V_{r}\right| S\right\rangle, \\
& B=\left\langle X\left|V_{r}\right| X\right\rangle .
\end{aligned}
$$

From Eqs. (3) one can derive two equations which give only $f_{1}$ and $f_{2}$ functions. Next, one can take expectation value of these equations with the use of $f_{1}$ and $f_{2}$ treated as variational functions. As a result one obtains general equation for expectation value of energy

$$
\sum_{i=0}^{5} a_{i}(E-A)^{i}=0
$$

where polynomial coefficients $a_{i}$ include average values of $V_{s}$ and kinetic energy operator.

The ETF of ground state for variational procedure is chosen in the form [7]:

$$
f=C \exp \left\{-\frac{1}{2}\left[\beta\left(\rho^{2}+\lambda z^{2}\right)\right]^{\delta}\right\},
$$

where $\beta, \delta$ and $\lambda$ are variational parameters.

One can see that for $\delta=1 / 2$ and $\lambda=1$ function (7) approaches $1 s$ hydrogen-like function and for $\delta=1$ it is Yafet et al. [8] function, whose transverse part presumably dominated by magnetic interaction at high fields, is taken in the form suitable for the free electron in magnetic field.

Numerical calculations have been done for GaAs and magnetic field from 0 to $100 \mathrm{kGs}$. In the first approximation we have taken $a=5.67 \times 10^{-2}$ a.u. Relation between $E-A$, where $E$ is energy of ground donor state and $A$ is defined by Eq. (4), and magnetic field is shown in Fig. 1. The parameter $A$ is a difference between $1 \mathrm{Ry}^{*}$, i.e. expected energy of donor ground state in absence of magnetic field, and energy $E-A$ for $B=0 \mathrm{kGs}$, therefore $A \cong 0.115 \mathrm{Ry}^{*}$ for $a=5.67 \times$ $10^{-2}$ a.u. Taking into account results obtained before [7] which very well agree with experimental data $[9,10]$ one can obtain relation of energy $A$ versus magnetic field. Figure 2 shows that effect of short range of donor potential slighly increases with magnetic field, i.e. when magnetic radius decreases. Functions of variational parame- ters versus magnetic field are presented in Fig. 3. 


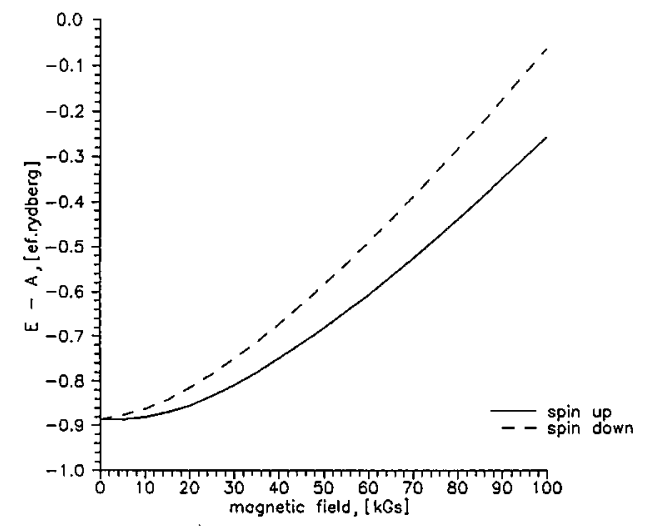

Fig. 1. Relation of $E-A$ versus magnetic field.
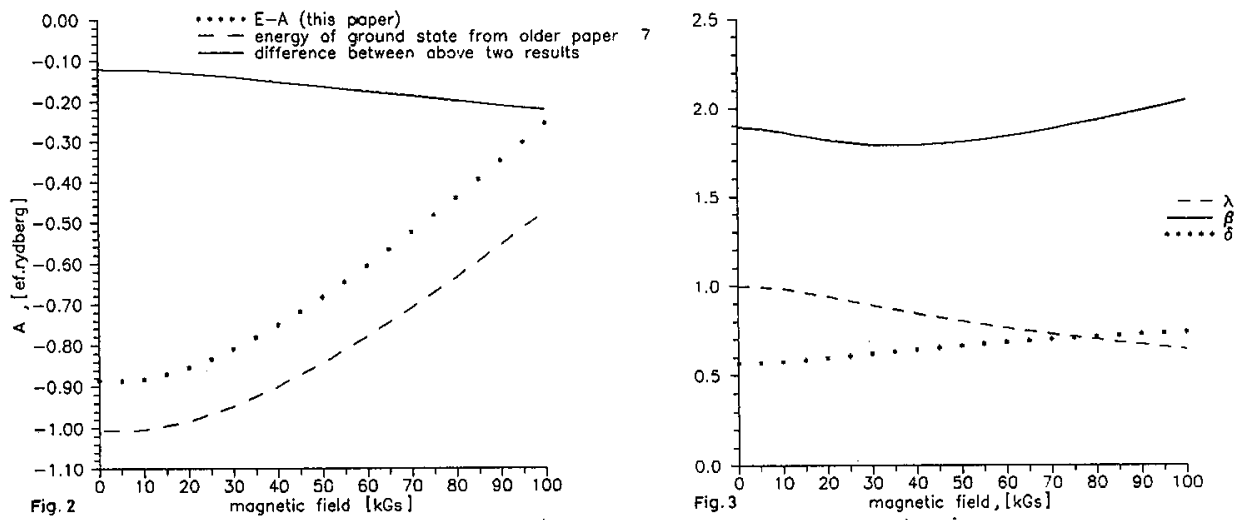

Fig. 2. Relation of A versus magnetic field.

Fig. 3. Relation of variational parameters versus magnetic field.

ETF fulfils crucial demands of approaching hydrogen-like function for zero magnetic field and Landau function for infinite magnetic field. Therefore our considerations could be applied for arbitrary magnetic field. We have also found that the energy of donor ground state is a continuous function of magnetic field. One can add that it is the first calculation of the donor ground state energy of III-V semiconductor in many band approach without taking any infinite energy contribution as small correction. Finally, this work can be treated as a preliminary step to the theoretical description of the impurity chemical shift.

\section{References}

[1] G. Dresselhaus, Phys. Rev. 100, 580 (1955).

[2] E.O. Kane, J. Phys. Chem. Solids 1, 249 (1957). 
[3] R. Bowers, Y. Yafet, Phys. Rev. 115, 1165 (1959).

[4] D.M. Larsen, J. Phys. Chem. Solids 29, 271 (1968).

[5] W. Zawadzki, J. Własak, in: Theoretical Aspects and New Developments in Magneto-Optics, Ed. J.T. Devreese, Plenum, New York 1980, p. 347.

[6] W. Trzeciakowski, M. Baj, S. Huant, L.C. Brunel, Phys. Rev. B 33, 6846 (1986).

[7] A. Obuchowicz, J. Własak, Acta Phys. Pol. A 79, 329 (1991).

[8] Y. Yafet, R.W. Keyes, E.N. Adams, J. Phys. Chem. Solids 1, 137 (1959).

[9] S.N. Holmes, R.A. Stradling, P.D. Wang, R. Droopad, S.N. Williams, Semicond. Sci. Technol. 4, 303 (1989).

[10] S.N. Holmes, R.A. Stradling, P.D. Wang, to be published. 